\title{
From human to mouse and back offers hope for patients with fibromyalgia
}

\author{
Kevin J. Tracey \\ Feinstein Institutes for Medical Research, Northwell Health, Manhasset, New York, USA.
}

\begin{abstract}
Fibromyalgia syndrome (FMS) is a highly prevalent, debilitating disease with heterogeneous symptoms of widespread pain and tenderness, fatigue, sleep disturbance, and impaired cognition. The cause of FMS is unknown, but the clinical constellation of symptoms and abnormalities in the neuroendocrine system, autonomic nervous system, and sleep implicate the nervous system in its pathogenesis. In this issue of the $\mathrm{JCl}$, Goebel, Krock, et al. identified antibodies from patients with FMS that produce FMS in mice by binding to satellite glial cells (SCCs), which envelope sensory neurons. Because antibodies harvested from patients with FMS, but not controls, stimulated SCCs to an activated state known to mediate chronic pain by augmenting neuronal activity, these findings reveal a pivotal role for autoreactive IgC in the pathophysiology of FMS. These important findings pave a pathway to study mechanism-based experimental therapeutics targeting IgC titers or antibody binding to SCCs underlying the neuroimmune dysfunction of FMS.
\end{abstract}

Pain, tenderness, nociceptors, and satellite glial cells

Patients with fibromyalgia syndrome (FMS) experience debilitating pain because their sensory neurons, termed "nociceptors," have heightened sensitivity to touch and pressure, as if their neurons are primed or supercharged to transmit pain signals in response to even minimal changes in the environment (1). The result is a characteristic fibromyalgia pain involving both sides of the body, with tender points that elicit exquisite pain and muscle spasms. A list of proposed, unproved etiologies for FMS pain include dysfunctional pain processing and structural changes in the spinal cord and brain, abnormal spontaneous nociceptor activity, sensitization of unmyelinated sensory and motor nerve fibers, depletion of epidermal neurons, and increases in proinflammatory cytokines. Although cytokines are known to increase sensitivity to pain and disrupt mood, a systematic review found that cytokine levels in FMS were not substantially different as compared to controls, except for IL-1 receptor antagonist, IL-6, and IL-8 (2). Another previously unexplored possibility for amped-up nociceptors in FMS points to satellite glial cells (SGCs), which are found wrapped around neuronal cell bodies in peripheral sensory, parasympathetic, and sympathetic ganglia. The SGCs closely adhere to the neuronal surface, residing across a narrow gap merely $20 \mathrm{~nm}$ wide, not much wider than a synaptic cleft (3). The result is a cellular community organized as neuron-SGC units that enable bidirectional molecular communication between the SGCs and neurons. Like other glial cells, SGCs release ATP, glutamate, TNF, other cytokines, and fractalkine, which have been implicated in stimulating nociceptors and producing pain behaviors. The cell bodies

\section{Related Article: https://doi.org/10.1172/JCl144201}

Conflict of interest: The author has declared that no conflict of interest exists.

of nociceptors providing sensory innervation to the body reside in the dorsal root ganglia (DRG), where SGCs encase the neurons. Chemical, inflammatory, or physical injury activates SGCs in sensory ganglia, leading to enhanced expression of the astrocyte marker glial fibrillary acidic protein (GFAP), downregulation of Kir4.1 channels, increased sensitivity to ATP, and release of cytokines. Together with other work, these observations suggest that activated SGCs can increase the sensitivity of nociceptors and amp up pain behaviors. But until the present work of Goebel, Krock, and colleagues published in this issue of the JCI, whether or how these SGC responses might be involved in FMS was unknown (4).

\section{Passive transfer of FMS IgG to mice}

Goebel, Krock, and colleagues purified IgG from patients with FMS and healthy control subjects and administered it daily

to mice by intraperitoneal injection. Within 24 to 48 hours the mice developed hypersensitivity to pressure in a standard Randall-Selitto paw-pressure test and marked increased sensitivity to noxious cold, which persisted for more than one week. Mice receiving this IgG also developed hypersensitivity to calibrated von Frey filaments, a widely used laboratory test of mechanical nociception. As occurs in human FMS, mice receiving FMS IgG also developed reduced grip strength as compared with mice treated with IgG from healthy controls. To assess sensitization of nociceptors, the authors prepared skin-saphenous nerve preparations from both groups of mice and observed that the mechanosensitive nociceptors from FMS IgG-treated mice produced action potentials in response to less force as compared with nerve preparations from mice receiving healthy control IgG. This important result indicates that FMS IgG mediates enhanced nociceptor firing even when disconnected from the CNS. Immunohis- 


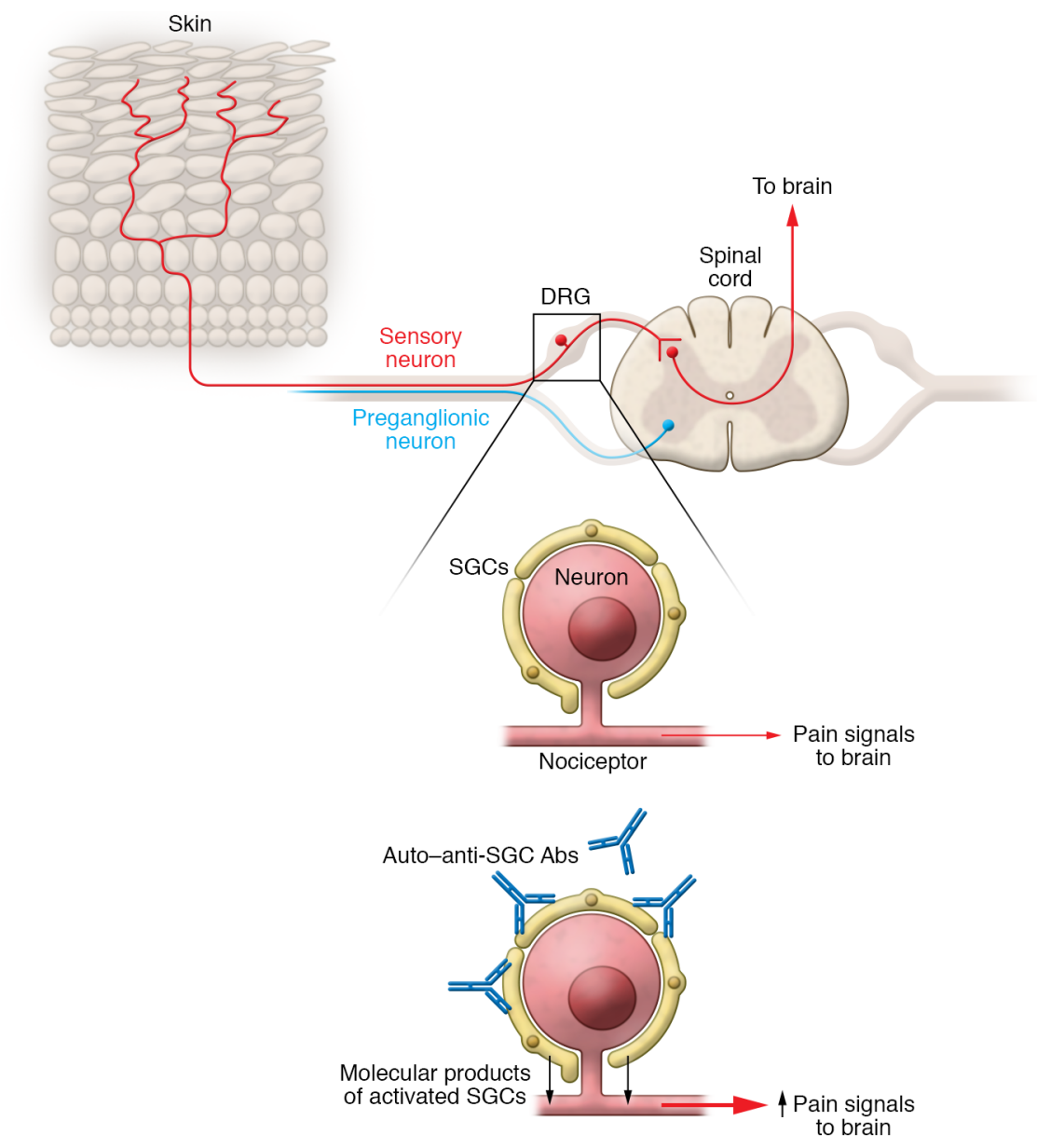

Figure 1. Model for autoreactive IgG in the pathophysiology of FMS. In dorsal root ganglia (DRC), SGCs encase sensory neurons that innervate the body. Autoreactive IgG antibodies that bind to SGCs may activate sensory neurons to amplify pain signals.

tochemical and Western blot localization revealed that FMS IgG accumulated in the DRG, not brain or spinal cord tissue, and this FMS IgG staining primarily localized to SGCs and to fiber tracts entering the DRG. The FMS IgG was also associated with evidence of enhanced SGC activation, because GFAP immunoreactivity and Gfap and $s 100 b$ gene expression were increased in DRG from mice injected with FMS IgG as compared with mice receiving control IgG. These changes in SGC activity in the DRG were specific, because no changes in astrocyte and microglia reactivity were found in the spinal cord. They further demonstrated that FMS IgG bound to cells in human DRG, including GFAP-immunoreactive cells and NF200-immunoreactive cells, suggesting FMS IgG can bind to both human SGCs and human sensory neurons. These results support their conclusion that “... IgG from FMS patients produces painful sensory hypersensitivities by sensitizing peripheral nociceptive afferents and suggest that therapies reducing patient IgG titers may be effective for fibromyalgia" (4).

\section{Clinical translational implications from humans to mice and back}

Outstanding clinical translational research linking clinical observations to basic research elevates equally both the clinical and scientific enterprises. The present work by Goebel and Krock et al. (4) is a shining, impactful example of how and why clinical translational research can and should be done. This tour de force study at once provides insight into a mysterious disease affecting millions of people and offers a solid and promising insight into just how to begin developing effective therapeutic strategies for a severe, chronic, uncurable illness. In this model, antibodies target SGCs to elicit FMS; there is no requirement to evoke CNS dysfunction or systemic inflammation, two of the previously most widely cited pathogenic mechanisms (Figure 1). And because the Goebel and Krock et al. results indicate that $100 \%$ of FMS donor IgG induced IgG dose-dependent FMS behaviors in mice, and FMS in mice resolved as the IgG levels waned, it seems likely that clinical trials may soon be possible using available therapies to reduce IgG titers, such as plasmapheresis or nonspecific immunoadsorption. Moreover, additional work may well lead to strategies that selectively reduce autoreactive IgG by antigen-specific adsorption after specific epitopes have been identified, which seems quite plausible. I expect this knowledge will also guide the development of mechanism-based experimental therapeutics to interfere with autoreactive antibody binding to SGCs or nociceptors, and to inhibit the functional activation of the SGCs. If that comes to pass, history may prove this work to be a classic case study for excellence in clinical translational research.

Address correspondence to: Kevin J. Tracey, Feinstein Institutes for Medical Research, 350 Community Drive, Manhasset, New York 11030, USA. Phone: 516.562.2813; Email: kjtracey@ northwell.edu.

1. Häuser W, et al. Review of pharmacological therapies in fibromyalgia syndrome. Arthritis Res Ther. 2014;16(1):201.

2. Uçeyler N, et al. Systematic review with meta-analysis: cytokines in fibromyalgia syndrome. BMC Musculoskelet Disord. 2011;12:245.

3. Hanani M, Spray DC. Emerging importance of satellite glia in nervous system function and dysfunction. Nat Rev Neurosci. 2020;21(9):485-498.

4. Goebel A, Krock E, et al. Passive transfer of fibromyalgia symptoms from patients to mice. JClin Invest. 2021;131(13):e144201. 